\title{
Rocket Observation of Suprathermal Electrons in the Energy Range 6.5-23 keV at Midlatitude
}

\author{
Tsuyoshi KoHNo \\ Department of Physics, Nagoya University, Nagoya, Japan
}

(Received February 8, 1973)

\begin{abstract}
Electrons of energies between 6.5 and $23 \mathrm{keV}$ have been observed from a sounding rocket with a thin-window $\left(37 \mu \mathrm{g} / \mathrm{cm}^{2}\right.$ polypropylene) proportional counter in the altitude range between $140 \mathrm{~km}$ and $326 \mathrm{~km}$ at the geomagnetic latitude of $20^{\circ} \mathrm{N}$, the $L$-value at the apogee being 1.17 .

The directional dependence of the electron flux with respect to the magnetic line of force indicates that these electrons consist of two components, the one peaked perpendicular to the line of force and the other being isotropic in the upper hemisphere. The former is considered to be geomagnetically trapped and the latter to be precipitating along the line of force. The flux ratio of the trapped to the isotropic components is $0.77 \pm 0.12$ and $1.7 \pm 0.2$ at $200 \mathrm{~km}$ and $300 \mathrm{~km}$, respectively. The isotropic component does not change between these altitudes, while the trapped component increases nearly twice as much. The observed intensities of the trapped and the isotropic electrons at $300 \mathrm{~km}$ are $(310 \pm 20)$ electrons $\cdot \mathrm{cm}^{-2} \cdot \mathrm{sec}^{-1} \cdot(4 \pi \text { sterad })^{-1}$ and $(27 \pm 4)$ electrons $\cdot \mathrm{cm}^{-2} \cdot \mathrm{sec}^{-1}$. sterad $^{-1}$, respectively.
\end{abstract}

\section{Introduction}

Since the discovery of the radiation belt the structure and the time variation have been extensively studied by sounding rocket and satellite observations. However, fewer experiments have been carried out on the structure of the lower edge of the inner radiation belt.

Because of the extremely large flux within the belt, those detector designed to measure the belt particles are apt to miss a small flux of particles below the lower edge of the radiation belt except at high latitudes where precipitating particles play an important role in auroral phenomena.

Some observations have indicated the existence of an appreciable flux of suprathermal particles below the lower edge of the radiation belt, which seems to persist steadily even in the geomagnetically quiescent period. Relevant publications are listed in an accompanying paper (HAYAKAWA et al., 1973b, hereafter referred to as ref. I).

Observations of these particles regarding their altitude dependence, pitch angle distribution, energy spectrum and time variations may reveal the behavior 
of particles in the magnetosphere in association with geomagnetic activities, the density variation in the exosphere and so forth, and may eventually lead to the understanding of the origin of these particles. Besides, such particles could be responsible, if not all, for the maintenance of the nocturnal ionosphere.

From quite a different point of view, the presence of suprathermal electrons presents a serious problem for cosmic X-ray observation because these electrons are the source of a large background when thin-window detectors are employed. Thus, it is also important for X-ray astronomy to know the flux of these electrons below the lower boundary of the belt.

In our previous flight (HAYAKAWA et al., 1973a, b), we observed electrons with energies of several $\mathrm{keV}$ at geomagnetic latitude $20^{\circ} \mathrm{N}$ in the altitude range $200-800 \mathrm{~km}$, corresponding to $L=1.17-1.25$. Although the absolute intensity was somewhat ambiguous because of the complexity in the detection efficiency, the electrons were found to be sharply trapped in the geomagnetic line of force at altitude above $500 \mathrm{~km}$ as shown in Fig. 7 in ref. I. The observed azimuthal dependence is approximately represented by the pitch angle distribution $\sin ^{n}$ $\alpha \mathrm{d}(\cos \alpha)$ with $n=8$, where $\alpha$ is the pitch angle of electrons with respect to the geomagnetic line of force.

MiYAZAKI et al. (1971) also observed at the same $L$-value a similar trapping feature of the electrons in the energy range $100-500 \mathrm{keV}$, the $n$-value being ten or larger.

On the other hand, LRL group (HILL et al., 1970) observed electrons of energies around $10 \mathrm{keV}$ preferentially directed along the geomagnetic line of force in the altitude range $100-150 \mathrm{~km}$ in their rocket observations from Kauai. $\left(22^{\circ} \mathrm{N}, 159.5^{\circ} \mathrm{W}, L=1.15\right)$. It can be shown that the observed azimuthal distribution is in very good agreement with that expected from an isotropic flux of electrons in the upper hemisphere with respect to the line of force.

Although the absolute flux of these electrons seems to be subject to a large time variation, we may conclude from the above observations that at higher altitudes, say above $500 \mathrm{~km}$, the electrons are mostly geomagnetically trapped, whereas in the lower altitude region $(100-150 \mathrm{~km})$ they are precipitating onto the atmosphere along the line of force. So far, observation of electrons in the intermediate altitude has been still lacking where the trapped and the precipitating components may become comparable. In the present paper we report on the observation of electrons in the intermediate altitude range where such transition actually occurs.

Our previous and present experiments have been performed with thinwindow proportional counters. In comparison with other detectors such as electron multipliers, the proportional counter has the following merits. First, a large effective area such as $100 \mathrm{~cm}^{2}$ is easily available. Second, the energy of each particle can be determined from pulse height measurement with reasonable resolution. Hence, we can measure a very small flux comparable to that of the 
cosmic ray particles. Furthermore, the proportional counter can be operative at all altitudes, even at low altitudes where windowless detectors can not be switched on.

\section{Instrumentation}

The detector consists of a thin-window proportional counter as shown schematically in Fig. 1. The window film thickness was 0.3 micron $\left(27 \mu \mathrm{g} / \mathrm{cm}^{2}\right)$ of polypropylene coated with $10 \mu \mathrm{g} / \mathrm{cm}^{2}$ carbon dag. Total effective area was $13 \mathrm{~cm}^{2}$.

Problems associated with the detection of electrons with thin-window proportional counters have been discussed in detail in ref. I. We shall summarize these problems briefly here. Incident electrons lose their energy through ionization loss inside the window film. Those electrons with sufficient energy can penetrate the film and deposit the residual energy inside the counter, giving rise to the pulse height equivalent to the measured energy. Because of the straggling in ionization loss and of Coulomb scattering, a given film thickness does not set a unique threshold for detection, but rather the detection probability is a function of incident electron energy. One can, however, define the effective threshold as to be the energy at which the detection probability is $50 \%$. As the electron energy increases above the effective threshold, the detection probability quickly approaches $100 \%$, and the energy loss in the film as well as its fluctuation also diminish. Monte Carlo calculation as described in ref. I yields the result for the film thickness of $37 \mu \mathrm{g} / \mathrm{cm}^{2}$ in this experiments, as shown in Figs. 2 and 3. From Fig. 3 by counting electrons above $5 \mathrm{keV}$ of the measured energy the effective threshold is about $6.5 \mathrm{keV}$.

The technical development of the proportional counters equipped with thin polypropylene window has been reported elsewhere (KAто et al., 1972).

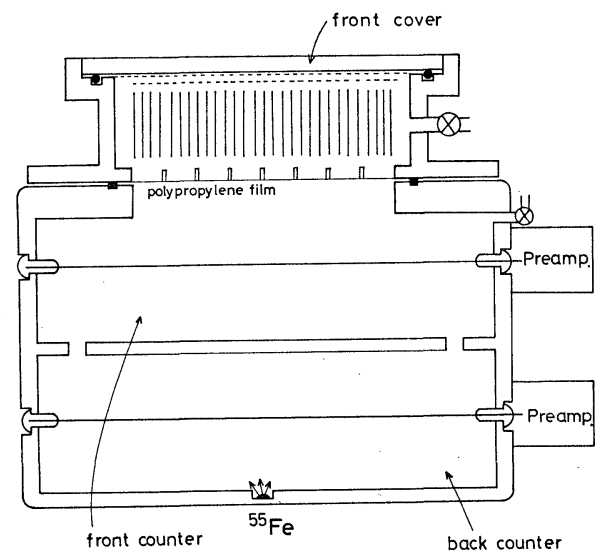

Fig. 1. Schematic view of the detector. 


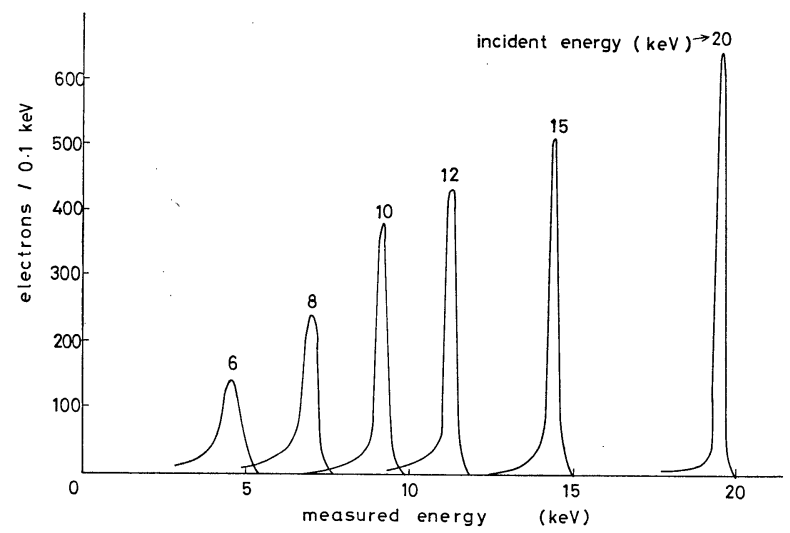

Fig. 2. Results of Monte Carlo calculation for the window thickness of $37 \mu \mathrm{g} /$ $\mathrm{cm}^{2}$. Curves represent measured energy distributions for several values of incident energy, each for 2000 incident electrons.

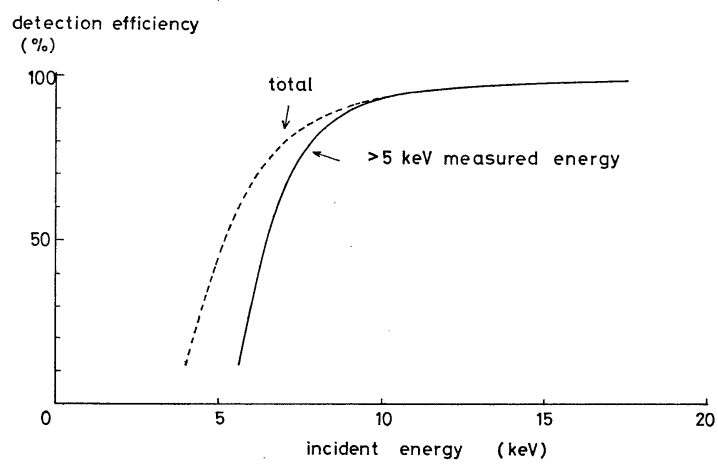

Fig. 3. Detection probability vs. incident energy of electrons derived from Fig. 2. The dashed curve indicates total transmission probability, whereas the solid curve indicates the detection probability for the requirement of the measured energy to be larger than $5 \mathrm{keV}$.

The gas flushing is usually required for the thin window counters to avoid the poisoning from ambient atmosphere due to the permeability of the window film. However, the gas flushing considerably complicates the detector system and the pre-launch operation. One particular feature of the present experiment is that the sealed-off counter is used. In order to be free from the contamination of the ambient air before launch, the collimator housing is sealed air-tight with a front cover plate. The collimator housing as well as the counter box are then filled with one atm. of the mixture of $90 \%$ argon and $10 \%$ methane. The gain drop of such a sealed-off counter during the storage is found to be less than a few per cent per week. The front cover plate is jetisoned two seconds after the nose cone ejection.

After the front cover ejection the counter gas leaks out slowly through the 
window film, thereby causing the gradual gain increase. For minimizing the gain shift during the flight the volume of the counter box was made as large compared to the window area as available. The sensitive volume of the counter was separated into two parts, i.e., the front counter and the back counter. The back counter continuously monitored the ${ }^{55} \mathrm{Fe} X$-rays $(5.9 \mathrm{keV})$, providing the in-flight calibration. Anti-coincidence between the front counter and the back counter removes substantial part of the background caused by the cosmic ray particles.

The collimator consists of parallel blades of anodized aluminium, which defined the field of view of $3^{\circ} \times 26^{\circ} \mathrm{FWHM}$ with the larger dimension parallel to the rocket axis. The direction of the field of view was perpendicular to the rocket axis. This collimator which we call electrostatic shutter functions to discriminate electrons from X-rays. A negative voltage of 900 volt was applied between the alternate blades so that the electrostatic field across the blades could reject electrons below $21 \mathrm{keV}$. The electrostatic shutter was switched on at 60 seconds after launch, and thereafter 2.4 seconds of shutter-on period and 5.0 seconds of shutter-off period were repeated throughout the flight. During the shutter-on period we expect to observe total non-electronic background, namely cosmic X-rays and the spurious background. Therefore, by subtracting the counting rate for the shutter-on period from that for the shutter-off period, the contribution of the electrons is to be obtained.

In the previous flight (ref. I) where a positive voltage was applied to a similar electrostatic shutter, we encountered a great many small pulses possibly generated by ambient plasma electrons which were accelerated by the leakage field. This time we screened the field by fine meshes.

The estimated contribution of cosmic diffuse X-rays is shown in Fig. 4. It is estimated to be about 5 percent of the background count rate observed before the nose cone ejection. As a matter of fact the observed counting rate increase at the removal of the front cover plate turned out to be negligibly small.

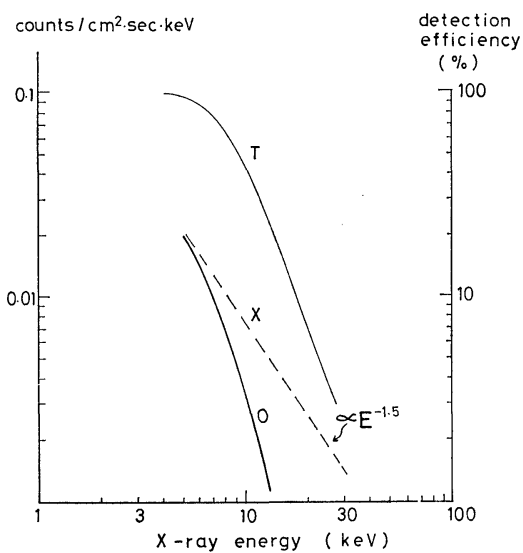

Fig. 4. Estimated contribution of cosmic X-rays. Thin line (T) expresses the detection efficiency of X-rays (ordinate on the right side). The dashed line $(\mathrm{X})$ indicates the intensity of diffuse X-rays entering in the field of view, employing the spectrum $11 E^{-1.5}$ photons $/ \mathrm{cm}^{2}$. sec.sterad $\cdot \mathrm{keV}$. Thick line $(0)$ is the resultant intensity of diffuse X-rays to be observed. 
Two IRIG channels were used to telemeter the data. Analog pulse height for each event of the front counter in the energy interval between $5-23 \mathrm{keV}$ was telemetered through one channel. The other channel contained in a time sharing basis the digital scaler output of the front counter, the voltage monitor of the electrostatic shutter and the analog pulse height of the back counter $\left({ }^{55} \mathrm{Fe}\right.$ X-rays).

\section{Rocket Flight and Experimental Performance}

The payload on board the sounding rocket, K-9M-40, was launched on Sept. 20, 1972 at 14:00 Japanese Standard Time or 5:00 U.T., from Kagoshima Space Center (geomagnetic latitude $20^{\circ} \mathrm{N}$ ). Solar and geomagnetic activity was quiet on and during a few days before the day of launch as seen from the $K p$ diagram in Fig. 5. The time sequence during the flight is shown in Table 1.

Useful data were obtained above $76 \mathrm{~km}$ altitude both for the ascent and the descent parts. The $L$-value at the apogee of $326 \mathrm{~km}$ altitude is 1.18 . The spin period of the rocket was $408 \mathrm{msec}$. The precession cone half angle was about $5.0^{\circ}$, and the effect of the precession was neglected in the analysis. The angle between the precession axis and the local geomagnetic line of force was approximately $30^{\circ}$. The position of the magnetic line of force and the plane perpendicular to it is shown in the rocket frame of reference in Fig. 6.

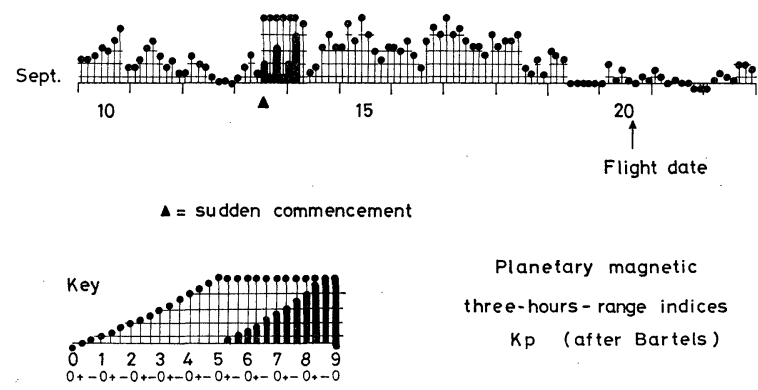

Fig. 5. Planetary magnetic three-hour-range indices $(K p)$ during several days before the day of launch (from Solar Geophysical Data, prompt reports, November 1972).

Table 1.

\begin{tabular}{lcc}
\hline \multicolumn{1}{c}{ Event } & Time after launch & Altitude \\
\hline Nose cone open & $52 \mathrm{sec}$ & $71 \mathrm{~km}$ \\
Front cover open & 54 & 76 \\
Electrostatic shutter switch on & 60 & 87 \\
Apogee & 293 & 326 \\
Termination of the data aquisition & 530 & 80 \\
\hline
\end{tabular}




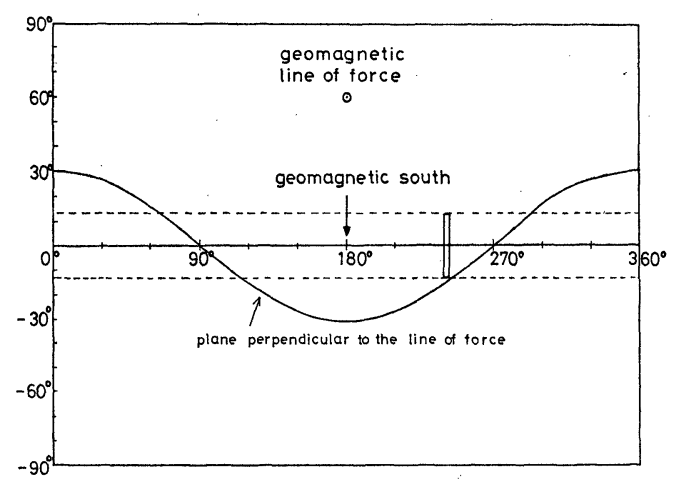

Fig. 6. Relation between the field of view and the plane perpendicular to the geomagnetic line of force in the rocket frame of reference. As the rocket rolls the counter field of view scans the belt covered by the dashed lines.

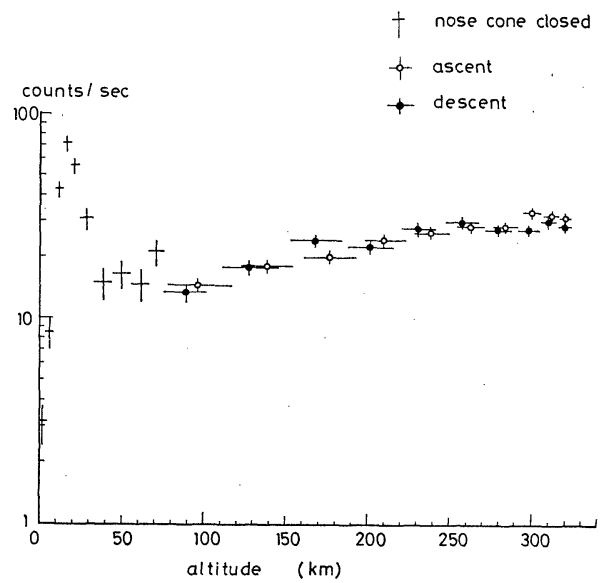

Fig. 7. Altitude dependence of total counting rate observed in ascent $\left(-\bigcirc_{-}-\right)$

and descent $\left(-\frac{1}{1}-\right)$ parts.

As the rocket rolls the field of view scans a belt along the great circle. There are two azimuthal angles at which the collimator axis crosses the plane perpendicular to the magnetic line of force.

Total gain increase during 530 seconds in flight as obtained from the back counter monitor of ${ }^{55} \mathrm{Fe} \mathrm{X}$-rays was found to be about $30 \%$ in agreement with that observed in the laboratory test. Pulse height data from the front counter are accordingly corrected. The observed counting rate during the electrostatic shutter-off period steadily increased from $110 \mathrm{~km}$ up to the apogee of $326 \mathrm{~km}$. As shown in Fig. 7 the data for the ascent part is in good agreement with that for the descent part. This clearly indicates the existence of a significant contribution of the electrons concerned which increase with altitude.

However, it turned out that the efficiency of electron rejection of the elec- 
trostatic shutter was not so good as we expected. The counting rate during the shutter-on period is actually smaller than that of shutter-off period, but also shows similar altitude dependence. The counting rate of cosmic-ray induced background will increase at most $10 \%$ due to the lowering cut off rigidity with the altitude, which will be substantially compensated by the diminishing rate of the background generated by the atmospheric $\gamma$-rays. Furthermore, as described in the later section, the counting rate observed in the shutter-off mode exhibits strong azimuthal anisotropy. Similar azimuthal anisotropy is also observed in the shutter-on mode which is quite unexpected from non-electronic background. Thus, we conclude that the electrostatic shutter did not remove more than $20 \%$ of the electrons concerned.

For the cause of this discrepancy we consider that the ambient plasma diffused through field-free gap into the collimator housing and partially neutralized the electric field across the collimator blades which were anodized and therefore insulated on their surfaces.

Thus the shutter-on period has not provided useful non-electronic data. Instead, we employ the data between $76 \mathrm{~km}$ (right after the front cover ejection) and $108 \mathrm{~km}$ as the background to be subtracted. As mentioned earlier, the counting rate in this period is within the statistical uncertainty the same as that before the nose cone ejection and the azimuthal anisotropy is absent.

Counts recorded in a number of successive spins are superposed for each appropriate interval for the analysis of data, and above-mentioned non-electronic background is subtracted therefrom. Data for the shutter-on period are not used for the later analysis.

\section{Experimental Results}

We derive (1) the azimuthal distribution, (2) the altitude dependence and (3) the energy spectrum of the electrons.

\subsection{Azimuthal distribution of the electrons}

After subtracting the background as mentioned in the previous section, we obtain the counting rate as a function of the azimuthal angle in the rocket frame of reference. The altitude range covered is divided into two intervals, 140-265 $\mathrm{km}$ and $265-326 \mathrm{~km}$. The results for these two altitude intervals are shown in Fig. 8(a) and (b), respectively. The arrow marked at $180^{\circ}$ in each figure indicates the direction of the geomagnetic south in the rocket frame of reference (see Fig. 6).

In both altitude intervals there appear two peaks symmetrically with respect to the magnetic south direction. It is, however, to be noticed that the counting rate minima in the north and the south directions are different, the northern minimum being deeper than the southern minimum. In the lower altitude range 

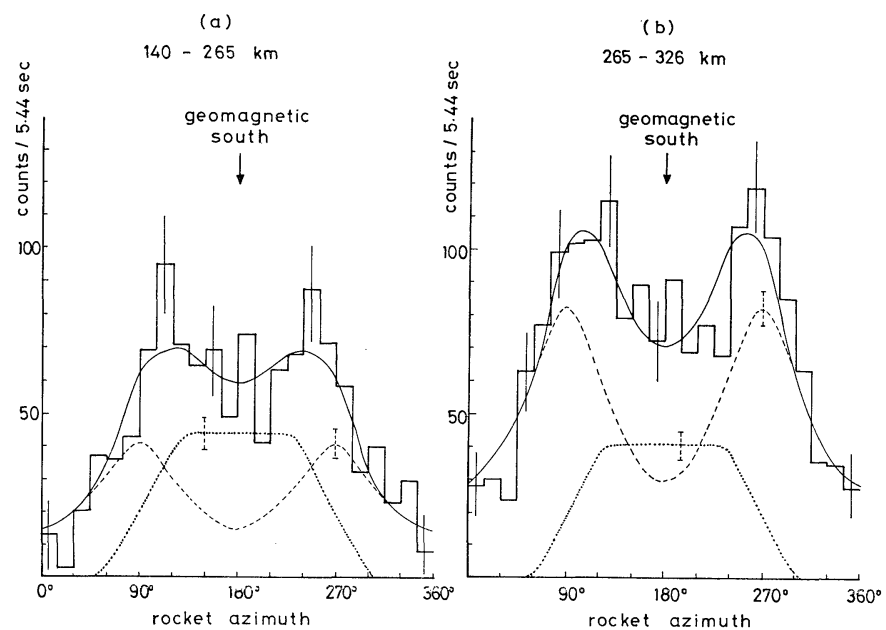

Fig. 8. The dependence of the electron counting rate on the rocket azimuth for two altitude ranges (a) $140-265 \mathrm{~km}$ and (b) $265-326 \mathrm{~km}$. The arrow marked at $180^{\circ}$ indicates the direction of the geomagnetic south in the rocket frame of reference. Curves represent calculated azimuthal distributions for the twocomponent model taking into account the collimator response function. The dotted curves are the distributions for the isotropic component in the upper hemisphere with respect to the magnetic line of force. The dashed curves are calculated for the trapped component employing the pitch angle distribution $\sin ^{8} \alpha \mathrm{d}(\cos \alpha), \alpha$ being the pitch angle with respect to the line of force. Solid curves show the sum of the dotted and dashed curves for the total electrons. The ratio of the respective components is determined by a least square method.

the southern minimum is not very pronounced. Whereas, as the altitude increases the two peaks grow rapidly and the southern minimum becomes more pronounced.

The presence of the two peaks can be understood in terms of geomagnetically trapped electrons which are gyrating predominantly in the plane perpendicular to the magnetic line of force. Since the field of view scans along a great circle, it crosses the plane of gyration which is another great circle twice per each rocket revolution (see Fig. 6). If all electrons were trapped by the magnetic line of force, two peaks, therefore, have to be located in due magnetic east and west directions and the two minima should have the same depth. The observed feature is different from what one expects for geomagnetically trapped electrons only.

We interpret the observed result in terms of two components of the electrons, namely the one being geomagnetically trapped and the other isotropic in the upper hemisphere with respect to the line of force. The latter component can be considered to be the precipitating electrons along the magnetic line of force. 
We further assume that the trapped component has a pitch angle distribution of the form $\sin ^{\mathrm{n}} \alpha \mathrm{d}(\cos \alpha)$, where $\alpha$ is the pitch angle with respect to the field line. Taking into account the response function of the collimator, the expected azimuthal distributions for respective components are calculated. We have adopted $n=8$ as taken in ref. I. The result is rather insensitive to the value of $n$ between 6 and 10, however. The ratios of the two components are determined by the least square method for two different altitude ranges. The results are also shown in Fig. 8 (a) and (b). As observed in these figures the composite azimuthal distributions are in good agreement with the observed results. The flux ratios of the trapped to the isotropic components $F_{\text {trap }} / F_{\text {iso }}$ are $0.77 \pm 0.12$ for $140-265 \mathrm{~km}$, and $1.7 \pm 0.2$ for $265-326 \mathrm{~km}$, respectively.

Moreover, we notice from the same figure that the isotropic component is almost independent of the altitude within the statistical errors, while the trapped component has increased by approximately a factor of two.

\subsection{Altitude dependence}

The altitude dependence of the total counting rate has been presented in Fig. 7. Subtracting the non-electronic background, the altitude dependence of the electrons in the range $6.5-23 \mathrm{keV}$ is shown in Fig. 9. Based on the two component model as described in section 4.1, the isotropic component is found to be almost independent of the altitude. The altitude dependence of the trapped component can be obtained approximately by subtracting the counting rates of the isotropic component which have been determined in the preceding section for two altitude intervals, as shown in the same figure.

In case that the ionization loss in the upper atmosphere is the predominant loss process for geomagnetically trapped electrons, the altitude dependence of the trapped component would in the first approximation be the same as that of the ionization life time. The latter is calculated by MAEDA (1965), and is re-

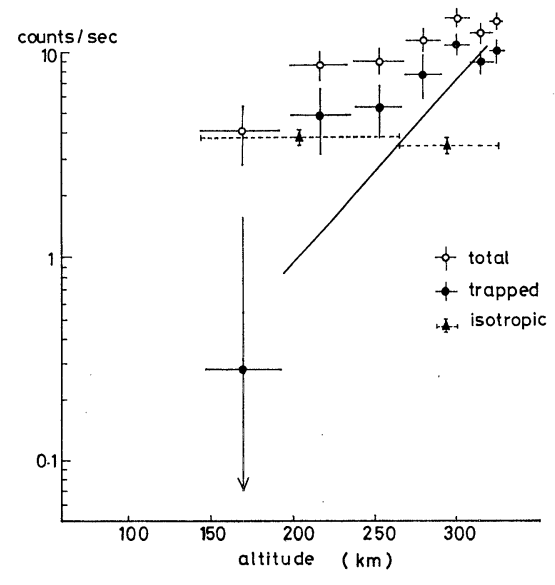

Fig. 9. The altitude dependence of the electrons after subtracting the non-electronic background (-Ó - - The altitude dependence of the trapped

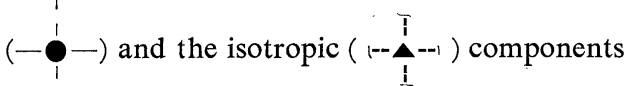
are also shown, based on the two-component model. 
produced in Fig. 9, with normalization to the average value of three observed points above $290 \mathrm{~km}$.

The observed altitude dependence of the trapped component seems slower than the above expectation. The implication will be discussed later.

\subsection{Energy spectrum of the electrons}

The observed spectra of the measured energy which is the energy deposited in the counter are presented in Fig. 10 (a) and (b) in two altitude ranges, 140 $265 \mathrm{~km}$ and $265-326 \mathrm{~km}$, respectively. In these figures we notice a general trend that the spectrum tends to flatten above $10 \mathrm{keV}$ in both altitude ranges, and that the spectra for the lower altitude range is somewhat softer than that for the higher altitude range. As shown in Fig. 11, the latter trend is also suggested by the difference of the two measured spectra, Fig. 10 (a) and (b). Namely, the increased part of the electrons between the two altitude ranges seems to have harder spectrum than the average one although the difference is not statistically significant. It is to be mentioned that the spectrum of Fig. 11 represents the measured energy spectrum of the increment of the trapped elec-
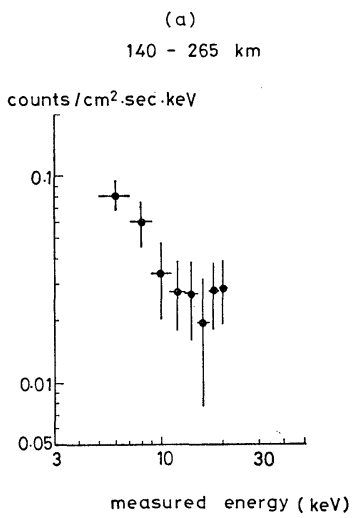

(b)

$265-326 \mathrm{~km}$

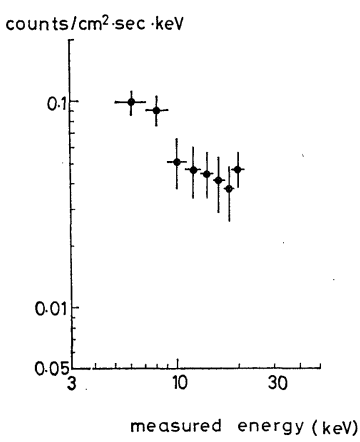

Fig. 10. The observed spectra of measured energy which is deposited in the counter for two altitude ranges, (a) $140-265 \mathrm{~km}$ and (b) $265-326 \mathrm{~km}$.

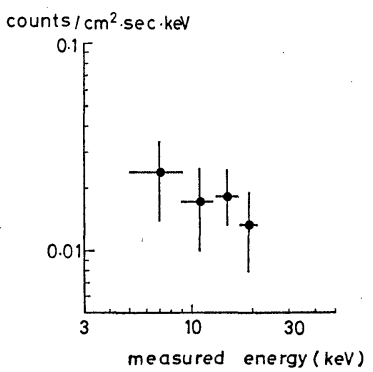

Fig. 11. Measured energy spectra of the increased part of the electrons between two altitude intervals (a) and (b), i.e., the difference between the two spectra in Fig. 10. 

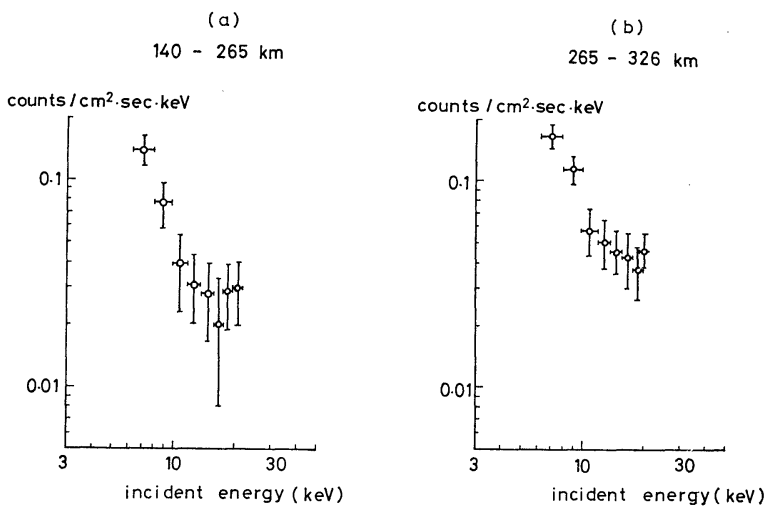

Fig. 12. Incident energy spectra of electrons which are obtained from the measured spectra Fig. 10(a) and (b) by correcting for the detection probability and the energy loss within the window film.

trons only, since the isotropic component does not change appreciably between the two altitudes concerned.

In order to derive the incident energy spectrum of electrons correctly, as discussed in Section 2, the measured spectrum has to be unfolded for the detection probability, fluctuation of the deposited energy and the resolution of the counter, each as a function of the energy. However, in view of rather poor statistics it is not worth following the exact procedure. As an approximate treatment, we only correct for the average energy loss in the window film and for the detection probability. The results of incident energy spectra derived in this way are shown in Fig. 12 (a) and (b). If one approximates the averaged incident spectra with a power function $E^{-\gamma}$, the exponent $\gamma$ is roughly 2 . However, as mentioned above, the spectrum tends to be harder with increasing energy.

\section{Discussions}

It has been shown in the previous section that the observed azimuthal distribution of electrons can be satisfactorily accounted for in terms of the twocomponent model, the trapped component and the isotropic component, in the altitude range $140-326 \mathrm{~km}$ covered in the present experiment. The trapped component steadily increases with altitude, whereas the isotropic component undergoes little altitude variation. Therefore, the trapped component will dominate at higher altitudes, while the isotropic component will become predominant at still lower altitudes.

In our previous experiment (ref. I) we observed that the electrons are almost all geomagnetically trapped above $500 \mathrm{~km}$. On the other hand LRL group 
(HILl et al., 1970) observed with a similar detector to ours that the electrons around $10 \mathrm{keV}$ are incident from general direction of the magnetic line of force at about $150 \mathrm{~km}$ altitude. In fact, their results of the azimuthal distribution can be very well fitted by an isotropic distribution in the upper hemisphere.

Thus, the results in three different altitude regions can be consistently understood in the frame of the two-component model. Indeed, the altitude region in the present observation turned out to be where the trapped and the isotropic component become comparable in intensity. It may be mentioned that the observed altitude variation by LRL group does not contradict the altitude independence of the isotropic component. Because the electrons around $10 \mathrm{keV}$ are considerably absorbed by traversing $100 \mu \mathrm{g} / \mathrm{cm}^{2}$ of atmosphere, attenuation of the flux of these electrons begins to be appreciable from $150 \mathrm{~km}$ downwards.

Formation of the two components can be explained in the following way. Suppose the injection of the electrons takes place at high altitude where the magnetic field is weaker than that in the altitude region of observation. Those electrons injected within a cone of a certain pitch angle will precipitate into the atmosphere without being reflected (loss cone), whereas those injected with larger pitch angles will be mirroring between conjugate mirror points, namely being geomagnetically trapped. For example, in case of the injection at the magnetic equator, the pitch angle of the loss cone is $48^{\circ}$. If the angular distribution of the electrons is isotropic at injection and as long as no scattering process is involved, the precipitating electrons maintain the isotropy while their pitch angle increase with increasing magnetic field downstream. Thus, these electrons at low altitudes form the isotropic component in the upper hemisphere with respect to the line of force. Furthermore, it is also evident that the intensity of this component is essentially constant until the atmospheric absorption becomes significant below $150 \mathrm{~km}$.

Consequently, the energy spectrum of the isotropic component is expected to be the same as the injection spectrum. It can be readily shown that the energy spectrum of the trapped component is one power harder than the injection spectrum, if the ionization loss is the decisive factor of the trapping life time. As mentioned in the previous section, the trapped component seems to show a harder spectrum than that of the total electrons. This is consistent with the above interpretation. However, in view of the insufficient statistics the conclusion should be reserved for further confirmation.

Regarding the altitude dependence of the trapped component, the observed result seems to indicate a slower increase than that expected from the trapping life time due to the ionization loss of the electrons. There may be two effects which will influence the altitude dependence, i.e., the effect of multiple scattering in the upper atmosphere and the conditions imposed on the electron injection. Further observations with increased statistics as well as theoretical con- 
siderations would be highly desirable, since these aspects have important bearings with respect to the origin of these electrons.

Finally, the absolute intensities of the trapped and the isotropic components are obtained. For the isotropic component the observed isotropic intensity is simply divided by the geometrical factor of the present detector $\left(0.3 \mathrm{~cm}^{2}\right.$ sterad $)$, which yields the intensity at $300 \mathrm{~km}$ altitude to be $(27 \pm 4)$ electrons $\cdot \mathrm{cm}^{-2} \cdot \mathrm{sec}^{-1}$. sterad ${ }^{-1}$ or, integrating over upper hemisphere, $(170 \pm 25)$ electrons $\cdot \mathrm{cm}^{-2} \cdot \mathrm{sec}^{-1}$. $(2 \pi \text { sterad })^{-1}$ for the incident energy range $6.5-23 \mathrm{keV}$. Whereas, the determination of the intensity of the trapped component requires the knowledge of the pitch angle distribution. Here, we tentatively derive the intensity of the trapped electrons by assuming the form $\sin ^{8} \alpha \mathrm{d}(\cos \alpha)$. The total intensity of the trapped electrons at $300 \mathrm{~km}$ in the same incident energy range is $(310 \pm 20)$ electrons. $\mathrm{cm}^{-2} \cdot \mathrm{sec}^{-1} \cdot(4 \pi \text { sterad })^{-1}$.

Direct comparison of the absolute intensities for different observation is rather ambiguous, because of the differences in the detector sensitivity, the altitude range, the observed angular distribution, etc. However, as clearly demonstrated in LRL observations, the absolute intensity of the electrons seems to undergo a large time variation. It would sensitively reflect the variation in the geomagnetic and atmospheric conditions. It may as well be different at various geographic locations. Repeated and preferably coordinated observations of these suprathermal electrons should yield useful information of the behavior of the particles in the lower magnetosphere and the exospheric atmosphere, and may eventually reveal the origin of these particles.

The author is indebted to Professor S. Hayakawa for his suggestion of this work. He also express his gratitude to Professor Y. Tanaka for his advices and encouragement throughout this experiment. Invaluable assistance of Dr. F. Nagase in the preparation of the experiment is gratefully acknowledged. The author also thanks the rocket launch crew of University of Tokyo for the successful operation.

\section{REFERENCES}

Hayakawa, S., T. Kato, T. Kohno, T. Murakami, F. Nagase, K. Nishimura, and Y. TANAKA, Existence of geomagnetically trapped electrons at altitudes below the inner radiation belt, J. Geophys. Res., 78, 2341-2343, 1973 a.

Hayakawa, S., T. Kato, T. Kohno, T. Murakami, F. Nagase, K. Nishimura, and Y. TANAKA, Suprathermal electrons near the lower edge of the radiation belt, J. Geomag. Geoelectr., 25, 113-129, 1973 b.

Hill, R.W., R.J. Grader, F.D. Seward, and J.P. Stoering, Soft particle flux above $130 \mathrm{~km}$ at midlatitude, J. Geophys. Res., 75, 7267-7271, 1970.

Kato, T., T. Kohno, F. Nagase, K. Nishimura, Y. Tanaka, and K. Yamashita, Thin polypropylene window proportioal counters for the observation of cosmic soft X-rays, Japan. $J$. Appl. Phys., 11, 1351-1356, 1972.

MAEDA, K., Further study on possible effects of the dynamo-electric field on electrons trapped in the magnetosphere, J. Geomag. Geoelectr., 17, 1-21, 1965.

Miyazaki, Y., H. TAkeuchi, T. Imai, and S. Watanabe, Pitch angle distribution of fast electrons, Bull. Inst. Space and Aeronautical Science, Univ. of Tokyo, 7, 133-144, 1971. 